\title{
Urban Feature Extraction on Simulated WorldView-2 Images
}

\author{
Camila Souza dos Anjos ${ }^{1,2}$ \\ Cláudia Maria de Almeida ${ }^{1}$ \\ Lênio Soares Galvão ${ }^{1}$ \\ Leila Maria Garcia Fonseca ${ }^{1}$ \\ ${ }^{1}$ National Institute for Space Research - INPE \\ São José dos Campos, Brazil \\ Email: \{camilasa, almeida, lenio\}@dsr.inpe.br \\ leila@dpi.inpe.br \\ ${ }^{2}$ Institute for Advanced Studies - IEAv \\ Brazilian Air Force \\ São José dos Campos, Brazil
}

\begin{abstract}
This work presents a procedure for simulating the eight multispectral bands of the WorldView-2 satellite by means of bands available in the airborne sensor HSS (Hyperspectral Scanner System). Both the original and simulated images were classified using the SAM (Spectral Angle Mapper) algorithm, aiming to discriminate intra-urban targets, namely: French tiles, asphalt/concrete, steel and aluminum tiles, bare soil, native arborous vegetation, and grass. The simulated images proved to be suitable for the detection of all aforementioned targets.
\end{abstract}

\section{INTRODUCTION}

The extreme heterogeneity of urban areas has showed to be one of the main causes of confusion in the classification of land cover and land use. In order to overcome such hindrances, many studies have now started to employ hyperspectral data in a collaborative way with high spatial resolution sensors [1]. Currently, high spatial resolution alone seems not sufficient for the investigation of urban areas. High spectral and radiometric resolutions are also required, since they are able to reduce conflicts in the extraction of urban features.

In the present work, a comparative analysis was carried out between classifications obtained from the original and the simulated WorldView-2 images, so as to evaluate the capacity for targets extraction of this high spatial resolution sensor containing eight spectral bands and of the HSS (Hyperspectral Scanner System), which is also a high spatial resolution sensor, but owning 37 spectral bands.

\section{A. Study Area}

The study area comprises part of São José dos Campos city, the seat of a municipality located in the eastern region of São Paulo state. The sensor line of flight owns $21 \mathrm{~km}$ of length and $1.6 \mathrm{~km}$ of width, covering part of important places in the city, like the airport, the Aerospace Technical Centre (CTA), the National Institute for Space Research (INPE), the President Dutra Road, and the REVAP Refinery Complex of the Brazilian Oil Company (Petrobras), as shown in Figure 1.

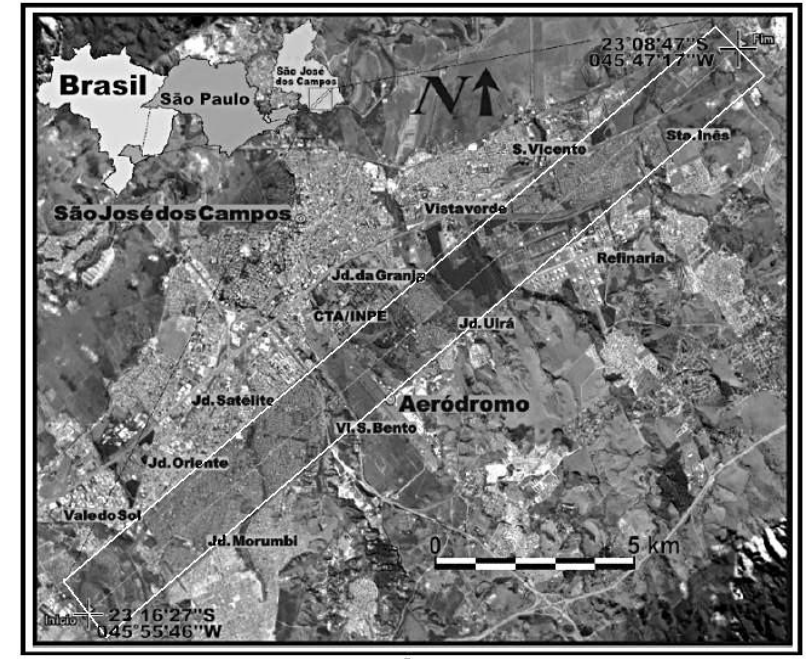

Figure 1. Line of flight in São José dos Campos adopted as study area. Source: Adapted from [2].

B. Sensors

\section{1) HSS (Hyperspectral Scanner System)}

The airborne sensor HSS, produced by the SensyTech company, contains 37 bands in the reflected VNIR and SWIR ranges, besides further 13 bands in the thermal spectral range, which have not been used in this particular work. The images have a radiometric resolution of 12 bits, with an interchangeable IFOV of 1.25 and $2.5 \mathrm{mrad}$ and an adjustable scanning frequency between 6.25 and $100 \mathrm{~Hz}$, which altogether may have an impact on the image spatial resolution and its signal-to-noise ratio.

\section{2) WorldView-2}

WorldView-2 is an optical orbital sensor produced by Digital Globe, which presents high spatial resolution and a differentiated spectral resolution in comparison with the 
traditional orbital high spatial resolution sensors. Its panchromatic and multispectral bands present approximately $0.5 \mathrm{~m}$ and $2.0 \mathrm{~m}$ of spatial resolution at nadir, respectively.

The images are produced with $11 \mathrm{bits}$ of radiometric resolution, and besides the conventional Blue, Green, Red, and Near Infrared bands, it renders available four other multispectral bands, namely Coastal Blue, Yellow, Red Edge, and an additional NIR band (Near Infrared 2).

\section{Objectives}

The main objective of this work is to evaluate the capacity of the WorldView-2 multispectral bands in discriminating urban targets, since the HSS sensor, which has also been commonly employed for this purpose, is no longer in operation.

For this end, WorldView-2 simulated images will be generated from HSS images. Both the original and the simulated images will be classified using the SAM (Spectral Angle Mapper) algorithm, so as to assess the suitability of such simulated images for the extraction of urban features.

\section{METHODS}

\section{A. Material}

The imaging with the HSS sensor was achieved in an aerial survey conducted from May 28 to June 2, 2006, with a flight height of approximately $1,070 \mathrm{~m}$ and an IFOV of $2.5 \mathrm{mrad}$, which resulted in an average spatial resolution of $2.7 \mathrm{~m}$ along the line of flight.

An HSS scene acquired on June 30, 2006 was used for the experiment reported in this work, comprising 37 bands in the VNIR and SWIR spectral ranges. The TIR bands have not been used, as previously stated.

\section{B. Pre-processing}

The initial step in the execution of this work was the image atmospheric correction. This procedure and the consequent generation of the surface reflectance image have been accomplished with the module FLAASH of ENVI 4.5.

\section{Simulation of WorldView-2 Bands}

For the bands simulation, the response function of the HSS and WorldView-2 sensors were used, which enabled to identify the exact spectral ranges of the HSS sensor that corresponded to each of the WorldView-2 (WV2) bands, and hence, calculate the overlap areas.

The method adopted for the bands simulation was based on the works of [3] and [4].

The calculation for the generation of each simulated band was done as in (1).

$$
S_{i}=\frac{1}{A_{S_{i}}}\left(\sum_{j=1}^{n} A_{b_{j}} N D_{j}+\frac{1}{n} A_{v_{i}} \sum_{j=1}^{n} N D_{j}\right)
$$

where:

$\mathrm{Si}=$ band to be simulated (WV2);

$\mathrm{ASi}=$ area of the response function curve to be simulated (WV2);

$\mathrm{j}=$ adjustment band (HSS);

$\mathrm{n}=$ number of adjustment bands (HSS);

$\mathrm{Abj}=$ area under the adjustment curve (area of the response function overlap) (HSS);

$\mathrm{AVi}=$ null area (area with no overlap of the simulated band by any other adjustment band);

$\mathrm{NDj}=$ digital number of the adjustment bands.

\section{Spectral Angle Mapper (SAM)}

The Spectral Angle Mapper (SAM) is a tool that allows mapping the spectral similarity between a given image and a reference spectrum. This algorithm determines the spectral similarity by calculating the angular difference between the two spectra, treating them as vectors in a multidimensional space, which number of dimensions is equal to the number of image bands [5].

Individual classifications were executed aiming at identifying on the HSS image as well as on the WorldView-2 simulated image the following targets: French tiles, asphalt/concrete, steel and aluminum tiles, bare soil, native arborous vegetation, and grass.

For each identified target, six samples were collected in the HSS image bands and in homologous points in the WorldView-2 simulated image bands, creating thus two spectral libraries related to the concerned target, one for each scene. The HSS scene was classified by SAM using the samples collected in the HSS image itself, regarding each of the targets of interest. The same procedure was applied to the WorldView-2 simulated image, producing six classifications for each scene.

Still in ENVI 4.5, the global accuracy was assessed for the WorldView-2 simulated image, using the classification conducted in the HSS image as the reference data. This accuracy index is obtained by summing the number of correctly classified pixels and dividing the final sum by the total number of existing pixels in the image.

\section{RESULTS AND DISCUSSION}

The simulated bands were visually analysed and presented a good agreement with the original WorldView-2 images of other regions than the study area. It is worth reminding that the simulation procedure does not alter the spatial resolution. In this sense, the simulated images present the same spectral characteristics of WorldView-2, but remain with the spatial resolution of the HSS sensor.

Figures 2 to 7 present the SAM classification results for each target, showing insets of the original HSS image and the WorldView-2 simulated image, with their respective classifications. 


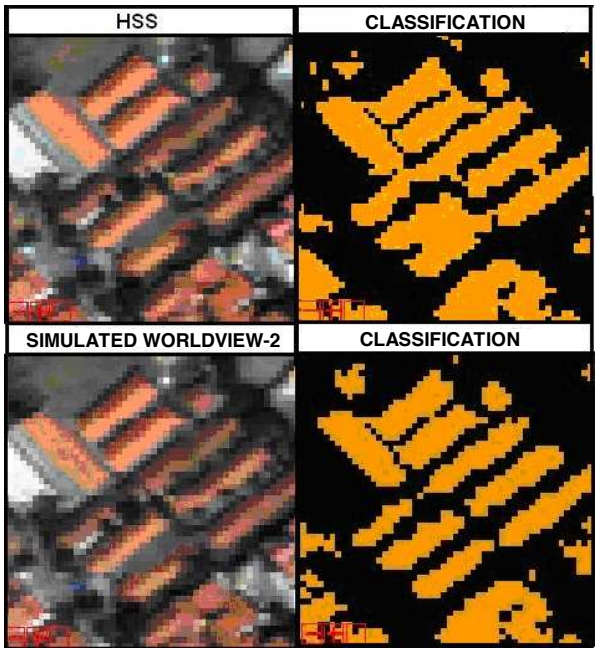

Figure 2. Insets of HSS and WV-2 simulated images and respective classification results for French tiles.

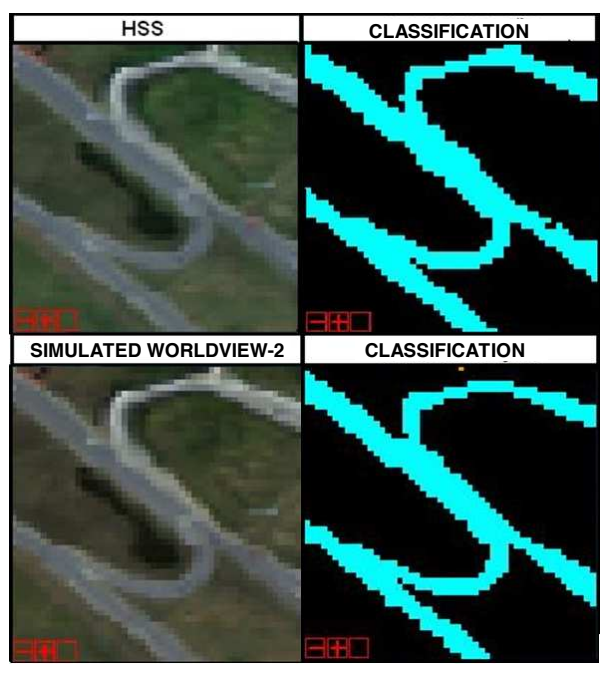

Figure 3. Insets of HSS and WV-2 simulated images and respective classification results for asphalt/concrete.

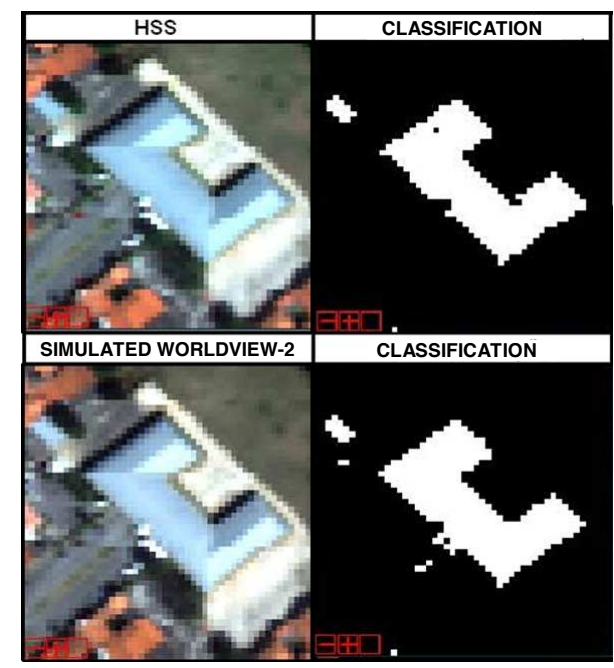

Figure 4. Insets of HSS and WV-2 simulated images and respective classification results for steel and aluminum tiles.

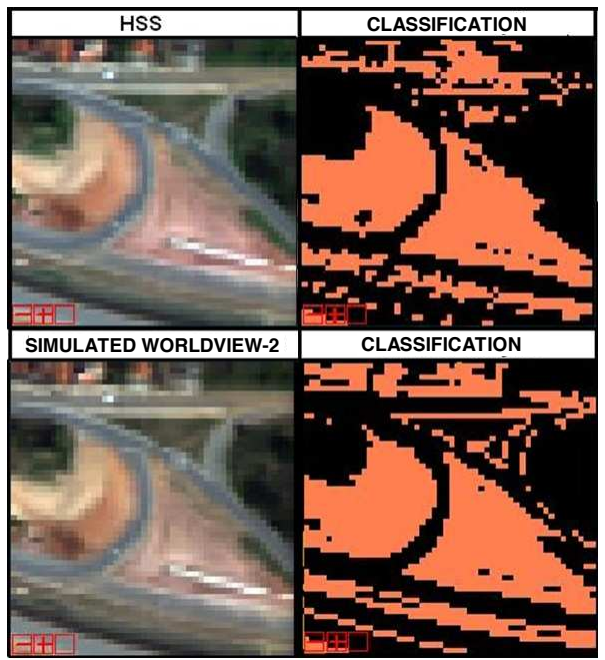

Figure 5. Insets of HSS and WV-2 simulated images and respective classification results for bare soil.

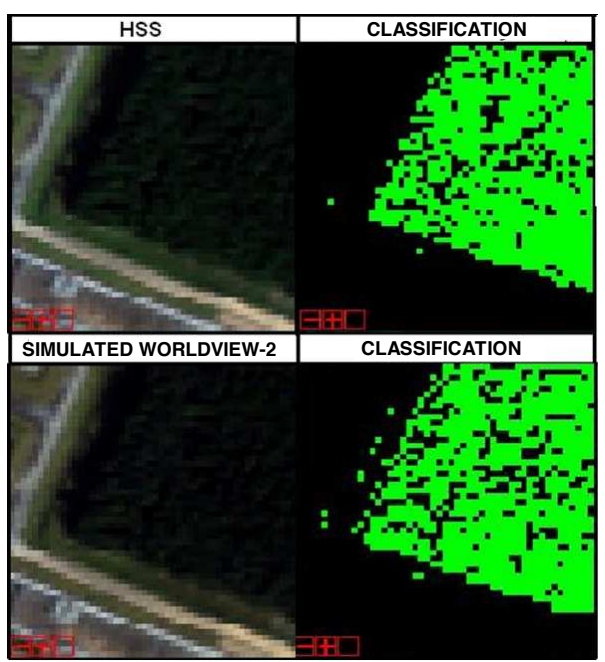

Figure 6. Insets of HSS and WV-2 simulated images and respective classification results for native arborous vegetation.

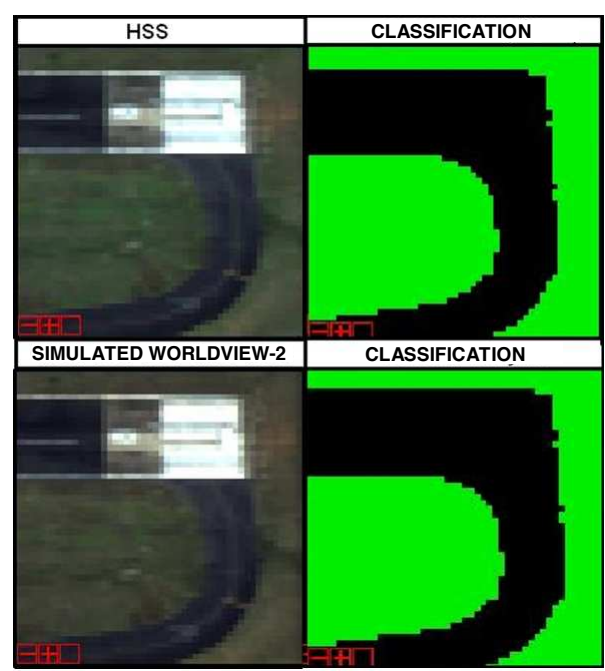

Figure 7. Insets of HSS and WV-2 simulated images and respective classification results for grass vegetation. 
The WorldView-2 simulated image was able to identify the targets of interest from the remaining targets found in the scene. A meaningful confusion was observed in the discrimination between bare soil and French tiles in both the HSS and the WorldView-2 simulated images, what is justifiable by the similar mineral composition of these targets.

As exposed previously, the classification accuracy of the WorldView-2 simulated image was calculated using the original HSS image classification as the reference data. Table 1 presents the global accuracy values for each target individually.

TABLE I. ClassificAtions ACCURACY.

\begin{tabular}{|c|c|}
\hline \multicolumn{1}{|c|}{ Classification } & Accuracy \\
\hline \multicolumn{1}{|c|}{ French Tiles } & $87.35 \%$ \\
\hline $\begin{array}{r}\text { Steel or Aluminum } \\
\text { Tiles }\end{array}$ & $98.52 \%$ \\
\hline Asphalt/Concrete & $82.35 \%$ \\
\hline \begin{tabular}{r|} 
Native Arborous \\
Vegetation
\end{tabular} & $91.45 \%$ \\
\hline Grass Vegetation & $72.05 \%$ \\
\hline Bare Soil & $77.68 \%$ \\
\hline
\end{tabular}

\section{CONCLUSIONS}

This work simulated the eight multispectral bands of the WorldView-2 satellite by means of bands available in the airborne sensor HSS (Hyperspectral Scanner System), executed an urban land cover classification in both images using the SAM algorithm, and finally, conducted an accuracy assessment to investigate the suitability of the two images for discriminating the urban targets of interest.
It can be observed that the two images differentiated well the concerned targets, although there has been confusion between bare soil and French tiles. In order to reduce such confusion, attributes based on bands ratio meant to enhance French tiles as proposed by [6] ought to be investigated in future works. The exploration of more sophisticated classifiers, like SVM, is as well envisaged.

Since the reported experiment used the original HSS image classification as the reference data for assessing the classification accuracy of the WorldView-2 simulated image, the authors recommend the acquisition of field spectra in further studies, so as to allow the evaluation of classification accuracy in both images, what would enable the selection of the most suitable one for extracting the features of interest.

Anyway, it is sensible to state that the WorldView-2 images can be used for identifying urban targets, even though it owns only eight bands, since the HSS - with its 37 bands - is no longer operational.

\section{REFERENCES}

[1] D. A. Roberts, D. A. Quattrochi, G. C. Hulley, S. J. Hook, R. O. Green, "Synergies between VSWIR and TIR data for the urban environment: An evaluation of the potential for the Hyperspectral Infrared Imager (HyspIRI) Decadal Survey mission" Remote Sensing of Environment, Vol. 117, 2012, pp. 83-101

[2] R. C. Moreira "Spectral Study of Urban Targets with Images from the HSS Sensor (Hyperspectral Scanner System)" Thesis (Phd in Remote Sensing) National Institute for Space Research, São José dos Campos, 2008, pp. 231

[3] N. D. A. Mascarenhas, G. J. F. Banon, L. M. G. Fonseca, " Simulation of a Panchromatic band by spectral linear combination of multispectral bands", In: Proceedings of International Geoscience and Remote Sensing Symposium, 1991, pp. 321-324

[4] B. M. G. Ribeiro, "Evaluation of WorldView-II Images for Mapping Urban Land Cover Using the InterIMAGE System” Dissertation (Msc. in Remote Sensing) National Institute for Space Research, São José dos Campos, 2010,pp. 151

[5] F. A. Kruse, A. B. Lefkoff, J. B. Boardman, K. B. Heidebrecht, A. T. Shapiro, P. J. Barloon, A. F. H. Goetz, "The spectral image processing system (SIPS) - interactive visualization and analysis of imaging spectrometer data" Remote Sensing of Environment, Vol. 44, 1993, pp. 145-163

[6] C. M. D. Pinho, M. E. Ummus, T. Novack, "Urban feature extraction in high spatial resolution images based on the investigation of the targets spectral behavior" Revista Brasileira de Cartografia. Vol. 63, 2011, pp. 619-627 